\title{
Toxicity effects of improved aged refuse on Tagetes patula and rhizosphere microbes
}

\author{
Fen $\mathrm{Hou}^{1}, \mathrm{Xu} \mathrm{Bi}{ }^{1}$, Ye $\mathrm{Yuan}^{1}$, Xihui $\mathrm{Wu}^{1}$, and Junjie $\mathrm{Du}^{2}$ \\ ${ }^{1}$ Shanxi University of Finance and Economics \\ ${ }^{2}$ Shanxi Normal University
}

June 13, 2021

\begin{abstract}
In this study, we examined the effects of different mass ratios of aged refuse on Tagetes patula and rhizosphere microbes. The results showed that chlorophyll content and activities of superoxide dismutase, catalase, and peroxidase in leaf tissue increased significantly in plants cultivated in soil:aged refuse mixtures compared with ordinary soil, whereas levels of malondialdehyde and protein carbonyl decreased significantly in soil:aged refuse mixtures. Microbial community analysis revealed that aged refuse is rich in a variety of rhizosphere microbes that contribute to pollutant degradation, although microbial diversity was found to be relatively low. Bacterial genera such as Ferruginibacter, Hymenobacter, unclassified_Gemmataceae, Longimicrobium, Tychonema CCAP 1459-11B, Gemmatirosa, and Rubellimicrobium tended to be enriched to a greater extent in ordinary soil compared with soil:aged refuse mixtures. Correspondingly, bacterial genera such as Emticicia, Caedibacter, Anaerosalibacter, Tumebacillus, Patulibacter, Oceanotoga, Dyadobacter, Chloroflexus, and Acidobacteria bacterium SCN 69-37, Polycyclovorans, tended to be enriched in mixtures with a higher proportion of aged refuse. Functional prediction analysis revealed that rhizosphere microbe functions changed markedly following the addition of aged refuse. These findings indicate that aged refuse may represent a source of environmental stress for plants and modifies the dominant bacterial composition of rhizosphere microbes. The combination of organic or inorganic pollutants, plant physiological stress responses, and rhizosphere microbial community composition may have potential cooperative or dynamic equilibrium relationships. With respect to identifying potential approaches to recycling aged refuse, it will be necessary to focus on selecting optimal mass ratios of aged refuse and ordinary soil to control contaminant exposure.
\end{abstract}

\section{Hosted file}

Manuscript.docx available at https://authorea.com/users/419581/articles/526041-toxicityeffects-of-improved-aged-refuse-on-tagetes-patula-and-rhizosphere-microbes 

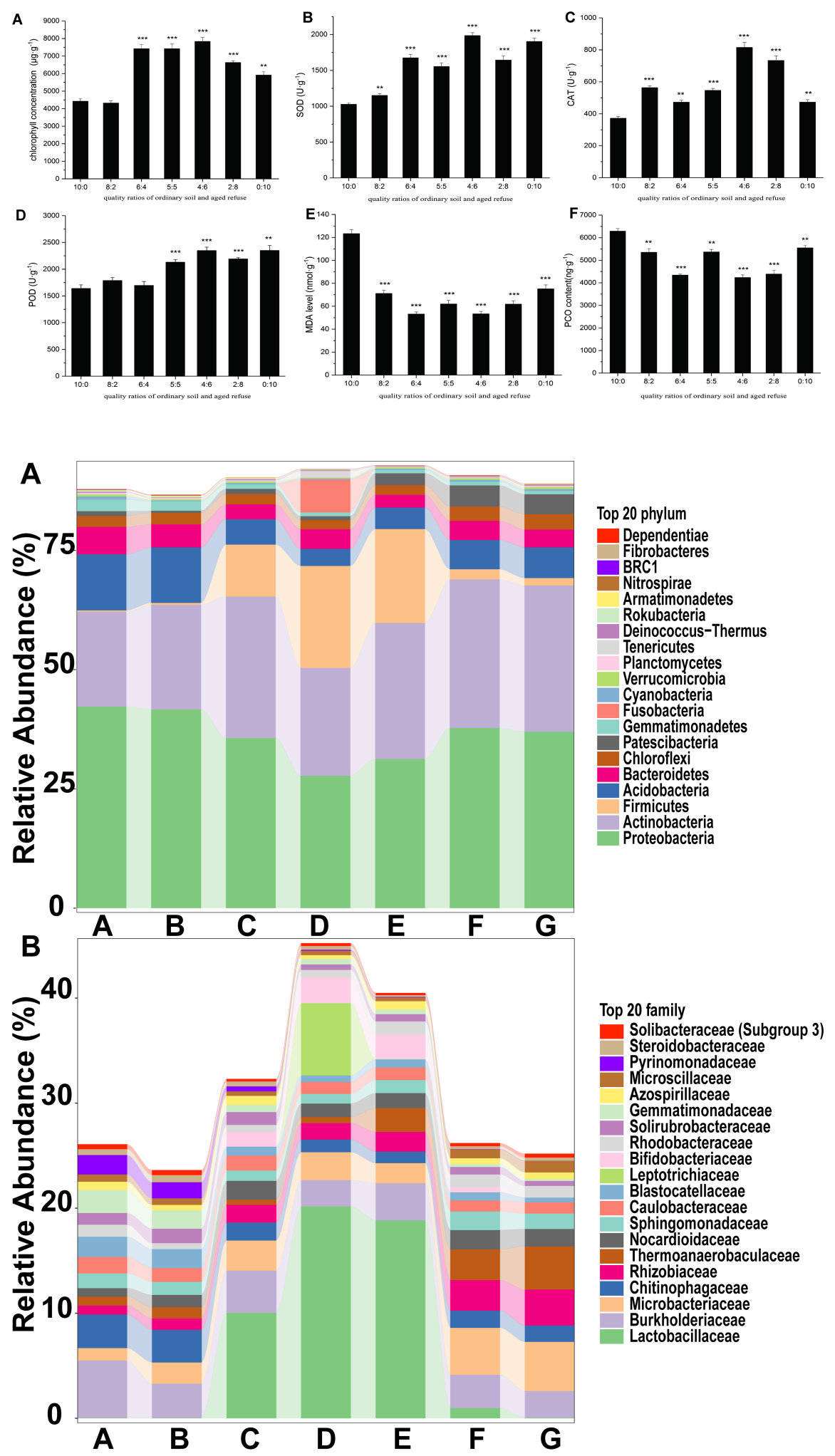

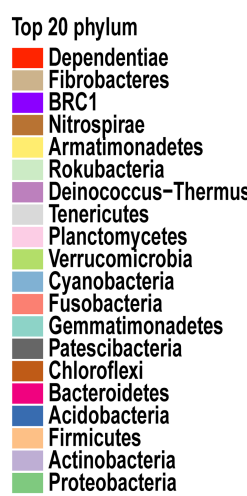

Top 20 family

Solibacteraceae (Subgroup 3)

Steroidobacteraceae

Pyrinomonadaceae

Microscillaceae

Azospirillaceae

Gemmatimonadaceae

Solirubrobacteraceae

Rhodobacteraceae

Bifidobacteriaceae

Leptotrichiaceae

Leptotrichiaceae

Baulobacteraceae

Caulobacteraceae

Sphingomonadacea

hermoanaerobaculaceae

Rhizobiaceae

Chitinophagaceae

Microbacteriaceae

Burkholderiaceae

Lactobacillaceae 

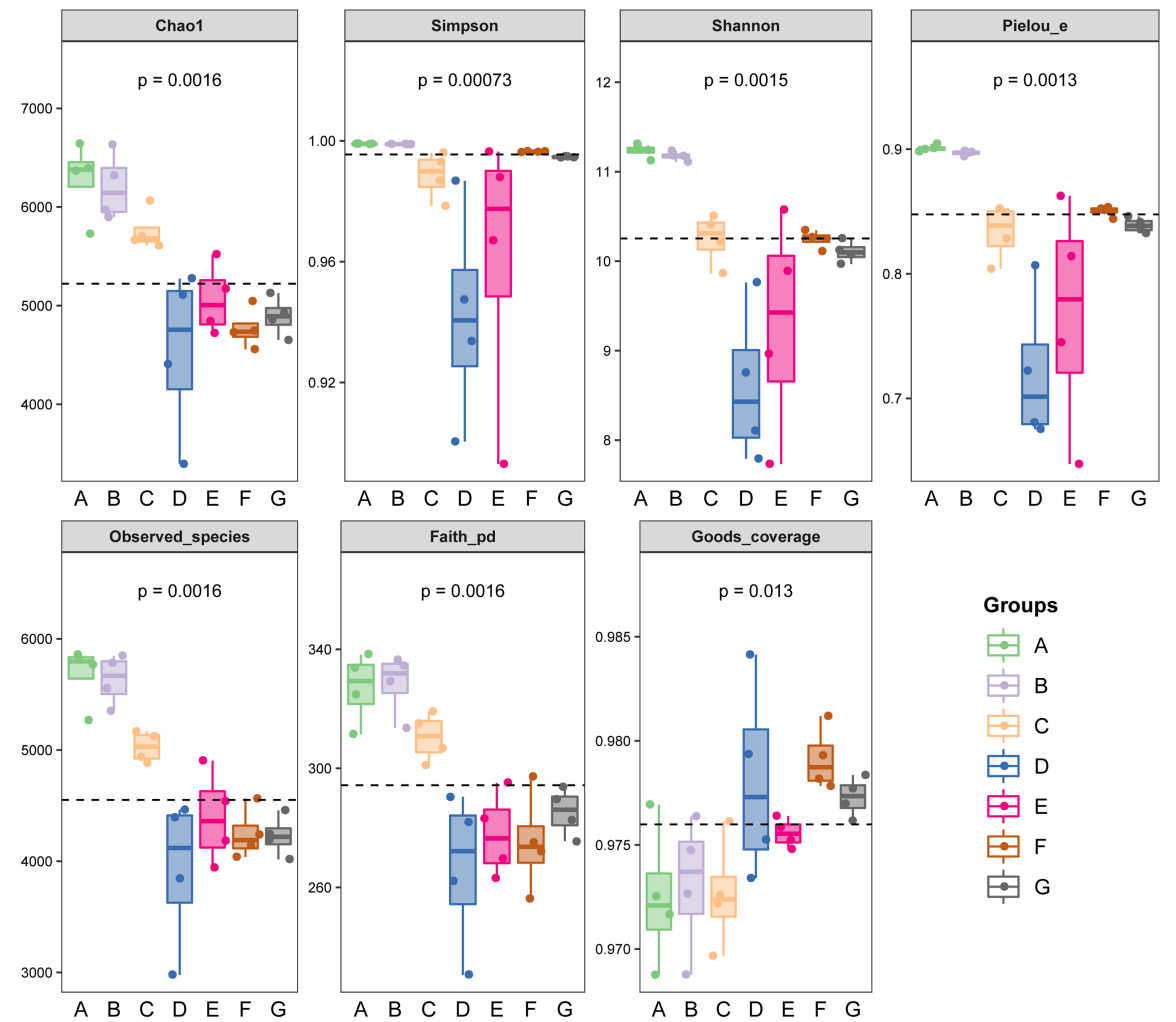

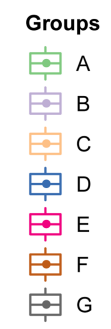




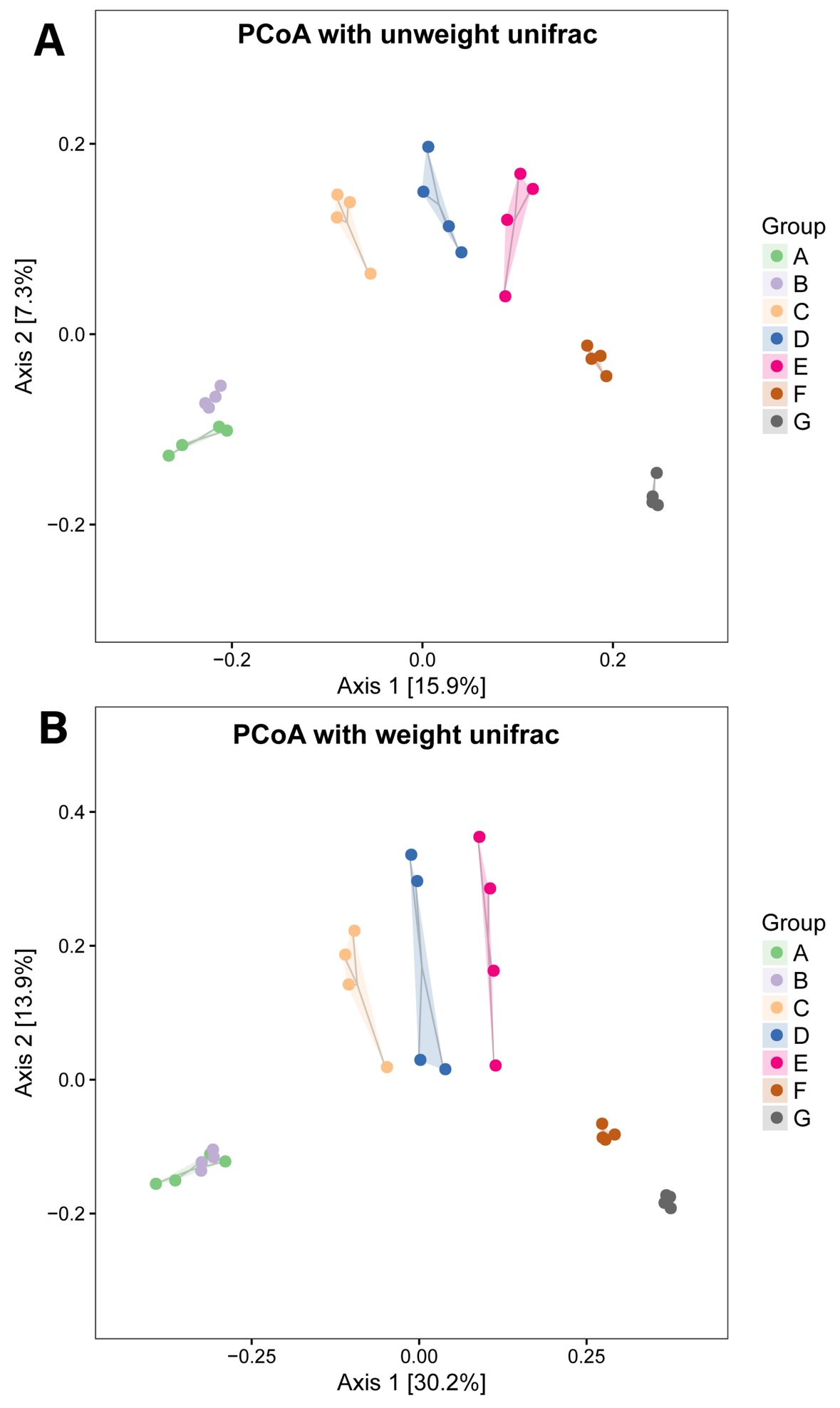



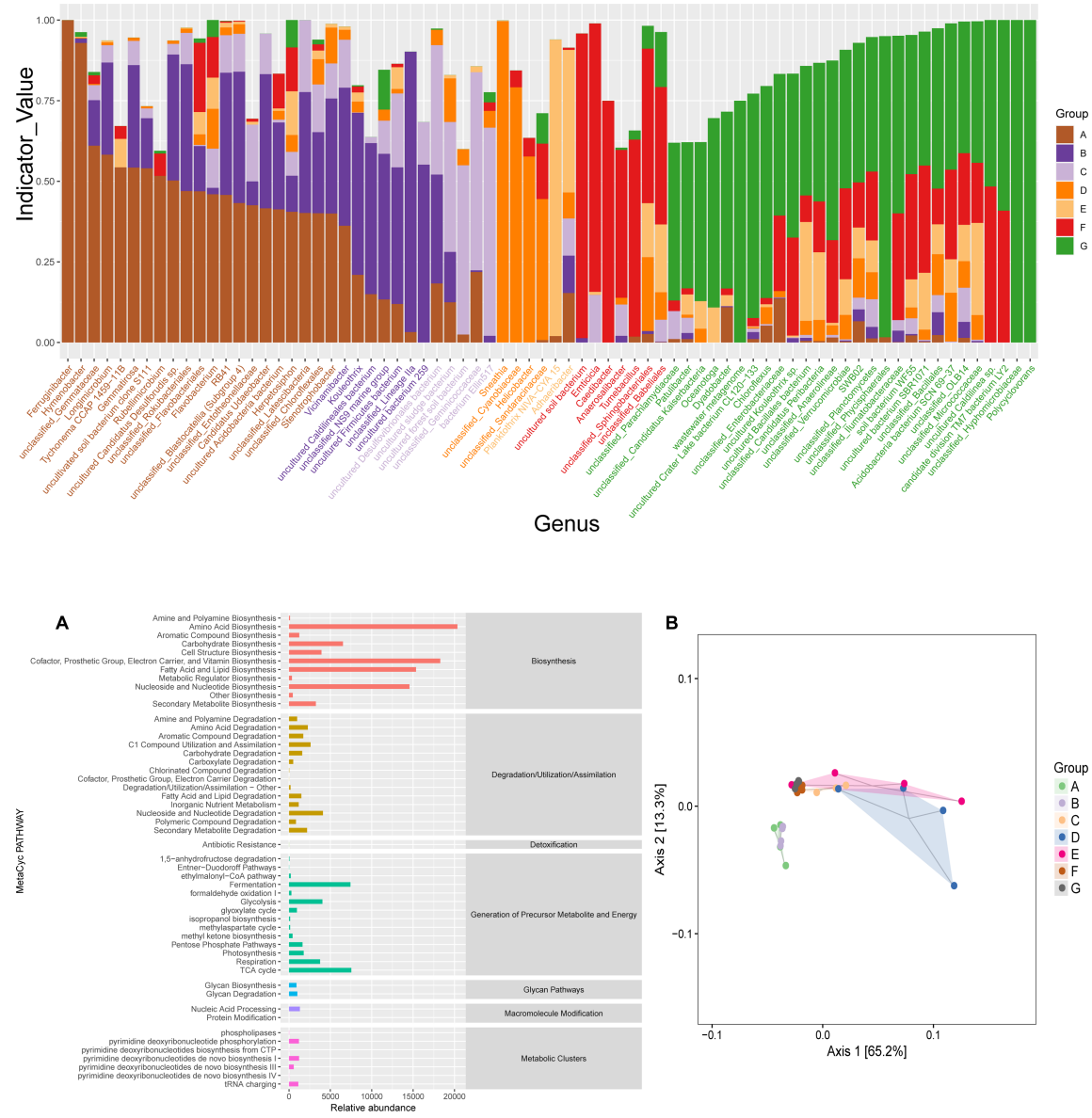